Received: 2014.10 .21 Accepted: 2014.11.18 Published: 2015.04 .27

\title{
Improved Preservation of Warm Ischemia- Damaged Porcine Kidneys after Cold Storage in Ecosol, a Novel Preservation Solution
}

Authors' Contribution: Study Design A Data Collection B Statistical Analysis C Data Interpretation D Manuscript Preparation E Literature Search F Funds Collection G
ABCDEF 1 Julia Kalenski

BCD 1 Elina Mancina

BCD 1 Pascal Paschenda

BCD 2 Christian Beckers

$A B C D 2$ Christian Bleilevens

$B C D$ 3,4 Lubomíra Tóthová

CDE 3,5 Peter Boor

ABCDEFG 1 Benedict Marie Doorschodt*

ACDEFG 1 René Hany Tolba*
1 Institute for Laboratory Animal Science \& Experimental Surgery, RWTH-Aachen University, Aachen, Germany

2 Department of Anesthesiology, RWTH-Aachen University, Aachen, Germany 3 Institute of Molecular Biomedicine, Comenius University, Bratislava, Slovakia 4 Center for Molecular Medicine, Slovak Academy of Sciences, Bratislava, Slovakia 5 Institute of Pathology \& Department of Nephrology, RWTH-Aachen University, Aachen, Germany
Corresponding Author: Source of support:

Background:

Material/Methods:

Results:

Conclusions:

MeSH Keywords:

Full-text PDF:
* Both authors contributed equally

René Hany Tolba, e-mail: rtolba@ukaachen.de

This study was supported by a grant from the START seed financing program of the RWTH-Aachen University (IA 691312) and research grants Q1 of the SFB/Transregio 57 "Mechanisms of organ fibrosis" and BO 3755/1-1 (both to PB) of the German Research Foundation ("Deutsche Forschungsgemeinschaft - DFG") and VEGA 1/222/14 of Ministry of Education, Science, Research and Sport of Slovak Republic

Ecosol, an extracellular-type, colloid-based preservation solution, has recently been introduced for washout, cold storage, and machine perfusion preservation of kidney grafts. Here, we assessed the efficacy of Ecosol compared to the widely used Histidine-Tryptophan-Ketoglutarate solution (HTK) for 24-h cold storage preservation of warm ischemia-damaged kidney grafts.

Before recovery, warm ischemia was induced by clamping the renal pedicle for $45-\mathrm{min}$. Thereafter, kidneys were washed-out and cold-stored for 24-h in Ecosol or HTK solution. Kidneys recovered without warm ischemia and cold-stored for 24-h in HTK served as controls $(n=5)$. Renal function and damage parameters were assessed during 1-h normothermic reperfusion using the isolated perfused porcine kidney model.

Renal function did not differ between Ecosol and controls and was significantly reduced in HTK compared to controls. Total output of urine was higher in Ecosol compared to HTK. Intrarenal resistance and urine protein concentrations in Ecosol were lower compared to HTK and equal to controls. In the Ecosol group, oxygen consumption during reperfusion was higher and reduced tissue lipid peroxidation products were detected compared to HTK.

The preservation quality of warm ischemia-damaged, cold-stored porcine kidneys was improved using the recently developed Ecosol preservation solution compared to HTK.

Kidney • Kidney Transplantation • Organ Preservation • Organ Preservation Solutions

http://www.annalsoftransplantation.com/abstract/index/idArt/892823

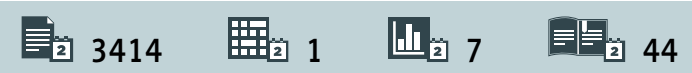




\section{Background}

Currently, more than 1 million patients worldwide are suffering from end-stage renal disease for which kidney transplantation is the only curative treatment [1]. The growing shortage of organs for transplantation has led to long waiting lists and a high mortality of patients awaiting transplantation [2] Insufficient availability of organs from brain dead and living donors has necessitated increased use of less than optimal organs, donated after cardiac death (DCD) or from extended criteria donors [3,4]. DCD kidney grafts have sustained warm ischemic damage, which causes tubular injury and impairment of glomerular filtration rate, resulting in higher incidences of delayed graft function [5]. Warm ischemic injury also promotes an uncontrolled deleterious innate and adaptive response [5]. Nonetheless, transplantation of DCD kidneys is associated with increased survival of patients who suffer from end-stage renal disease and are enrolled on transplant waiting lists [6,7].

Cold static storage (CS) is today still the most widely used organ preservation method because of its simplicity and low operational and logistical costs [8], although hypothermic machine perfusion is associated with reduced rates of delayed graft function compared to CS [9]. Since the introduction of the first CS solution in 1969 by Collins [10], several preservation solutions have been developed to combat the detrimental effects of warm and cold ischemia. Histidine-TryptophanKetoglutarate (HTK), Celsior and University of Wisconsin (UW) solution are currently widely used for preservation of kidney grafts, with comparable post-transplant outcomes in most clinical trials $[8,11,12]$. However, CS of DCD organs using the current clinically applied preservation solutions is associated with up to $73 \%$ incidence of delayed graft function $[8,13]$. The preservation solution is of vital importance to increase the organ's tolerance to warm and cold ischemic damage and to mitigate reperfusion injury $[14,15]$. Several attempts have been made to develop new preservation solutions designed to improve early graft function, which to date have failed to reach wide clinical acceptance [16-19].

Recently, a novel preservation solution has been developed, named Ecosol (TX Innovations BV, Maastricht, The Netherlands) containing multiple buffering agents, antioxidants, amino acids, and vitamins to provide substrates for supporting the cell metabolism, which, although profoundly reduced, is active during hypothermic storage [20-22]. Ecosol is based on the synthetic colloid polyethylene glycol (PEG) and several impermeants (Table 1) to achieve the oncotic pressure required to prevent extravasation of the solution during washout of the graft prior to CS as well as during hypothermic machine perfusion preservation $[19,23]$. As a constituent of organ preservation solutions, PEG is associated with immunomodulatory effects, reduction of lipid peroxidation, and stabilization of proteins against degradation [24]. When added to extracellular-type solutions (high $\left[\mathrm{Na}^{+}\right]$, low $\left[\mathrm{K}^{+}\right]$ratio), PEG has been shown to improve the preservation quality of kidney and liver grafts compared to the intracellular-type solutions HTK and UW $[25,26]$. However, extracellular-type solutions facilitate translocation of $\mathrm{Na}^{+}$into the cell under hypothermic conditions, thereby creating an osmotic gradient that promotes potentially lethal cell swelling [27]. Ecosol solution is an extracellular-type solution; therefore, several impermeants are embodied to counteract cell swelling.

Since successful preservation of DCD organs today remains a challenging endeavor, we aimed to assess the feasibility of Ecosol solution for 24-h CS preservation of 45-min warm ischemia-damaged kidney grafts in comparison to HTK using the isolated perfused porcine kidney model (IPPK).

\section{Material and Methods}

\section{Experimental protocols}

The experiments were performed in accordance with the German legislation governing animal studies following the 'Guide for the Care and Use of Laboratory Animals' (NIH publication, $8^{\text {th }}$ edition, 2011) and the Directive 2010/63/EU on the protection of animals used for scientific purposes (Official Journal of the European Union, 2010). Official permission was granted from the governmental animal care and use office (LANUV Nordrhein-Westfalen, Recklinghausen, Germany).

In the Ecosol and HTK groups, kidneys were subjected to 45-min warm ischemia and consequently washed-out and cold-stored in their respective solutions ( $n=5$ per group, Figure $1 \mathrm{~A}$ ). Kidneys recovered without warm ischemia, washed-out and cold-stored for 24-h in HTK served as a negative control group $(n=5)$.

Female German Landrace pigs with $46 \pm 1 \mathrm{~kg}$ body weight (BW, mean \pm SEM) from a disease-free barrier breeding facility were housed in fully air conditioned rooms $\left(22^{\circ} \mathrm{C}\right.$ room temperature, $50 \%$ relative humidity) and allowed to acclimatize to their surroundings for a minimum of 7 days and fasted for $12 \mathrm{~h}$ before surgery with free access to water. The animals were premedicated with $8 \mathrm{mg} / \mathrm{kg}$ BW azaperone (Stresnil ${ }^{\circledR}$, Janssen-Cilag $\mathrm{GmbH}$, Neuss, Germany), $15 \mathrm{mg} / \mathrm{kg}$ BW ketamine (Ceva $\mathrm{GmbH}$, Duesseldorf, Germany), and $10 \mathrm{mg}$ atropine ( $1 \mathrm{ml} / 1 \%$ atropine sulfate, Dr. Franz Köhler Chemie GmbH, Bensheim, Germany) administrated intramuscularly. General anesthesia was induced by $2 \mathrm{mg} / \mathrm{kg}$ BW propofol (Fresenius Kabi Deutschland $\mathrm{GmbH}$, Bad Homburg, Germany) administered intravenously, followed by intubation and mechanical ventilation with 1.5 Vol\% isoflurane (Forene ${ }^{\circledR}$, Abbot $\mathrm{GmbH} \&$ Co. KG. Wiesbaden, Germany) and oxygen with continuous intravenous infusion 
Table 1. Composition of HTK and Ecosol (mM).

\begin{tabular}{|c|c|c|c|}
\hline Component & & HTK & Ecosol \\
\hline Colloid & PEG 35.000 & - & 5.4 \\
\hline \multirow[t]{7}{*}{ Impermeants } & Sodium gluconate & - & 37.1 \\
\hline & Magnesium gluconate & - & 12.0 \\
\hline & Calcium gluconate & - & 2.3 \\
\hline & Lactobionic acid & - & 12.0 \\
\hline & Trehalose & - & 4.0 \\
\hline & Raffinose & - & 1.7 \\
\hline & Mannitol & 30.0 & - \\
\hline \multirow[t]{5}{*}{ Buffers } & HEPES & - & 16.8 \\
\hline & Sodium bicarbonate & - & 0.4 \\
\hline & Potassium phosphate & - & 2.2 \\
\hline & Sodium citrate & & 3.1 \\
\hline & Histidine & 198.0 & 10.0 \\
\hline \multirow[t]{2}{*}{ Anti-oxidants } & Glutathione & - & 12.0 \\
\hline & Taurine & - & 36.8 \\
\hline \multirow[t]{5}{*}{ Energy substrates* } & Glucose & - & 6.9 \\
\hline & Pyruvate & - & 1.8 \\
\hline & Adenine & - & 6.0 \\
\hline & Tryptophan & 2.0 & 1.5 \\
\hline & Ketoglutarate & 1.0 & - \\
\hline Electrolytes & {$[\mathrm{Na}+] /[\mathrm{K}+]$} & $15 / 9$ & $124 / 13$ \\
\hline Osmolarity $(\mathrm{mOsm} / \mathrm{L})$ & & 310 & 395 \\
\hline Viscosity $\left(\mathrm{cP}\right.$ at $\left.21^{\circ} \mathrm{C}\right)$ & & 1.8 & 2.8 \\
\hline $\mathrm{pH}\left(21^{\circ} \mathrm{C}\right)$ & & $7.0-7.2$ & 7.4 \\
\hline
\end{tabular}

* Ecosol contains the vitamins ascorbic acid, biotin and the amino acids arginine, carnitine, cysteine, glutamic acid, glutamine, glycine and ornithine.

A

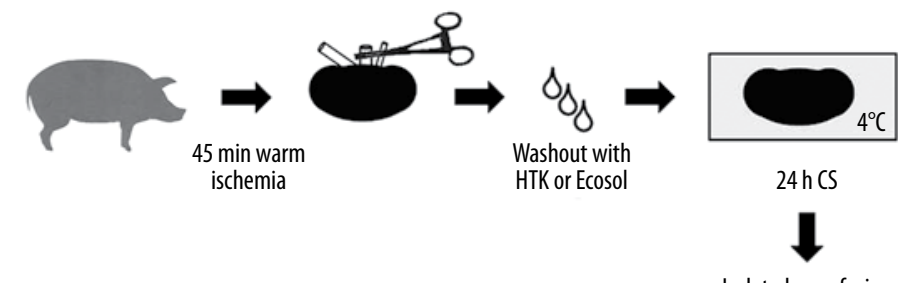

B

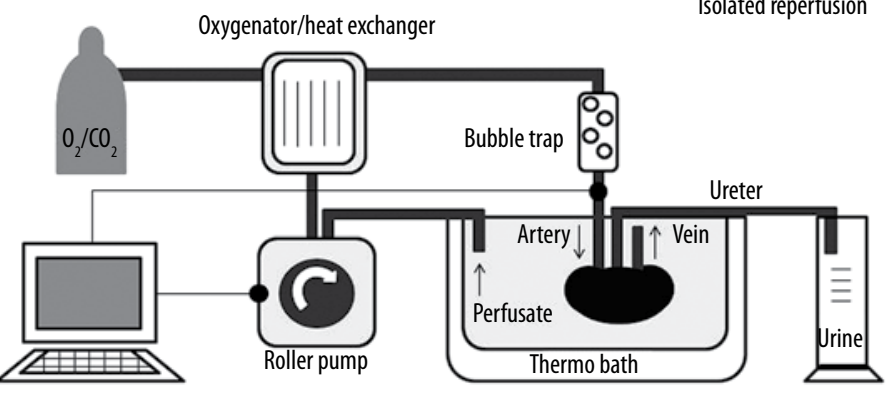

Figure 1. (A, B) Schematic of the experimental design and isolated perfused porcine kidney model. 
of $0.02 \mathrm{mg} / \mathrm{h} / \mathrm{kg}$ BW fentanyl (KG Rotexmedica GmbH, Trittau, Germany). After a midline laparotomy, both kidneys were explanted with or without induction of warm ischemia. Directly after recovery, $1 \mathrm{~L}$ of whole blood was collected into citratephosphate-dextrose bags (Fenwal Inc., Illinois, USA) and stored at $4^{\circ} \mathrm{C}$ for ex-vivo reperfusion. The animals were euthanized by IV administration of $1 \mathrm{ml} / \mathrm{kg}$ BW pentobarbital (Narcoren ${ }^{\circledR}$, MERIAL GmbH, Hallbergmoss, Germany).

\section{Kidney recovery}

For induction of warm ischemia, both the renal artery and vein were clamped for 45 min followed by recovery, weighed, and washed-out immediately in case of controls or after $45 \mathrm{~min}$ warm ischemia via the renal artery. For washout, either 500 $\mathrm{ml}$ of cold HTK or Ecosol solution was used at a hydrostatic pressure of $100 \mathrm{~cm} \mathrm{H}_{2} \mathrm{O}$. Thereafter, kidneys were weighed and stored in their respective preservation solutions at $4^{\circ} \mathrm{C}$ for $24 \mathrm{~h}$

\section{Reperfusion}

As reperfusion medium, modified Krebs-Henseleit-Buffer (9.6 $\mathrm{g} /$, Sigma-Aldrich Chemie $\mathrm{GmbH}$, Steinheim, Germany), calcium chloride (0.37 g/l, Sigma-Aldrich Chemie GmbH), 8.4\% sodium hydrogen carbonate ( $25 \mathrm{ml} / \mathrm{l}$, Fresenius, Germany), creatinine (1 mmol/l, Sigma-Aldrich Chemie $\mathrm{GmbH}$ ), heparin (3000 IU/l, Ratiopharm GmbH, Ulm, Germany), and Fibrisol (3 g/l, Muscalla, Vierheim, Germany) were dissolved in $800 \mathrm{ml}$ water for injection (Ampuwa, Fresenius Kabi AG, Bad Homburg, Germany) and $200 \mathrm{ml}$ of autologous non-leucocyte-depleted whole blood was added to the mixture.

After CS, kidneys were weighed and placed into an organ reservoir filled with $1 \mathrm{~L}$ reperfusion medium pre-warmed to $38^{\circ} \mathrm{C}$ using a heating bath (HAAKE DC30, W13, Thermo Electron $\mathrm{GmbH}$, Karlsruhe, Germany) and pressure controlled reperfused for $60 \mathrm{~min}$ at a pre-set mean arterial pressure of 85 $\mathrm{mmHg}$ (Figure 1B). The reperfusion medium was circulated by a computer controlled pulsatile roller pump (ISMATEC ${ }^{\circledR}$, MPC Standard, Gladburg, Switzerland) through an oxygenator (Hilite ${ }^{\circledR} 2400$ LT, MEDOS, Stolberg, Germany) and bubble trap to the renal artery and exited the kidney freely into the organ reservoir. Pressure (MLT844, AD Instruments $\mathrm{GmbH}$, Spechbach, Germany) and flow sensors (ME2PXL1072 sensor, TS410 flow meter module, Transonic Systems Inc., Ithaca, NY, USA) were connected to the renal artery and data collected using a data acquisition system (PowerLab 8/30, AD Instruments $\mathrm{GmbH}$, Spechbach, Germany). Renal blood flow (RBF), mean arterial pressure (MAP), and temperature were continuously recorded and stored using Lab Chart 7 software (AD Instruments $\mathrm{GmbH}$ ). The intrarenal resistance (IRR) was calculated as MAP/RBF/100 g.
The reperfusion medium was continuously oxygenated with carbogen ( $95 \%$ oxygen $/ 5 \%$ carbon dioxide), achieving an arterial partial oxygen pressure $\left(\mathrm{pO}_{2}\right)$ of over $500 \mathrm{mmHg}$ throughout the reperfusion period. Arterial and venous $\mathrm{pO}_{2}$ and $\mathrm{pH}$ levels were measured at 5, 15, 30, 45, and 60 min using a blood gas analyzer (ABL 725, Radiometer GmbH, Willich, Germany). Renal metabolic activity was approximated by calculation of oxygen consumption using arterial and venous $\mathrm{pO}_{2}$ values $\left(\left(\mathrm{p}_{\mathrm{a}} \mathrm{O}_{2}-\mathrm{p}_{\mathrm{v}} \mathrm{O}_{2}\right)\right.$ $\times \mathrm{RBF} /$ kidney weight). Urine samples were collected separately at 5, 15, 30, 45, and 60 min during reperfusion for determination of sodium, creatinine, and urine protein concentrations and the total output of urine was recorded. The perfusate volume was replenished every $15 \mathrm{~min}$ to compensate for the excreted urine volume. Venous perfusate samples were taken at 5, 15, 30, 45, and $60 \mathrm{~min}$ and analyzed for sodium and creatinine levels. Using venous perfusate and urine levels, creatinine clearance (urine creatinine $\times$ urinary flow/plasma creatinine), and fractional excretion of sodium (urinary sodium $\times$ plasma creatinine)/(plasma sodium $\times$ urinary creatinine) $\times 100 \%$ were calculated.

\section{Neutrophil gelatinase-associated lipocalin}

Urinary levels of the acute tubular injury marker neutrophil gelatinase-associated lipocalin (NGAL, Kit 044, BioPorto Diagnostics, Gentofte, Denmark) were determined at the end of reperfusion using enzyme-linked immunosorbent assay (ELISA) according to the manufacturer's instructions. The absorbance was detected at $450 \mathrm{~nm}$ using a microplate reader (Infinite M200, Tecan Austria GmbH, Grödig, Austria).

\section{Oxidative status}

For assessment of the oxidative status, frozen tissue samples taken after reperfusion were homogenized in ice-cold phosphate-buffered saline $(10 \% \mathrm{w} / \mathrm{v})$, then centrifuged for $5 \mathrm{~min}$ at $4000 \mathrm{~g}$ and the supernatants were stored at $-20^{\circ} \mathrm{C}$. Concentrations of reduced glutathione (GSH) and oxidized glutathione (GSSG) were determined as described previously and the ratio of GSH to GSSG calculated [28]. Also, thiobarbituric acid reactive substances (TBARS) as a byproduct of lipid peroxidation was determined and expressed in $\mu \mathrm{mol}$ per gram protein [29]. The renal tissue protein content was assessed using bicinchoninic acid assay (BCA, Fermentas, Vilnius, Lithuania). All measurements were performed using a Saphire II spectrofluorometer (Tecan Austria GmbH, Grödig, Austria).

\section{Histology}

After reperfusion, kidney tissue slices were fixed in $10 \%$ formalin for $24 \mathrm{~h}$ and embedded in paraffin. Four-micron sections were stained either with Acid Fuchsin Orange G (AFOG) or with the periodic acid-Schiff reagent and counterstained with hematoxylin (PAS) and fully digitalized using a whole-slide scanner 

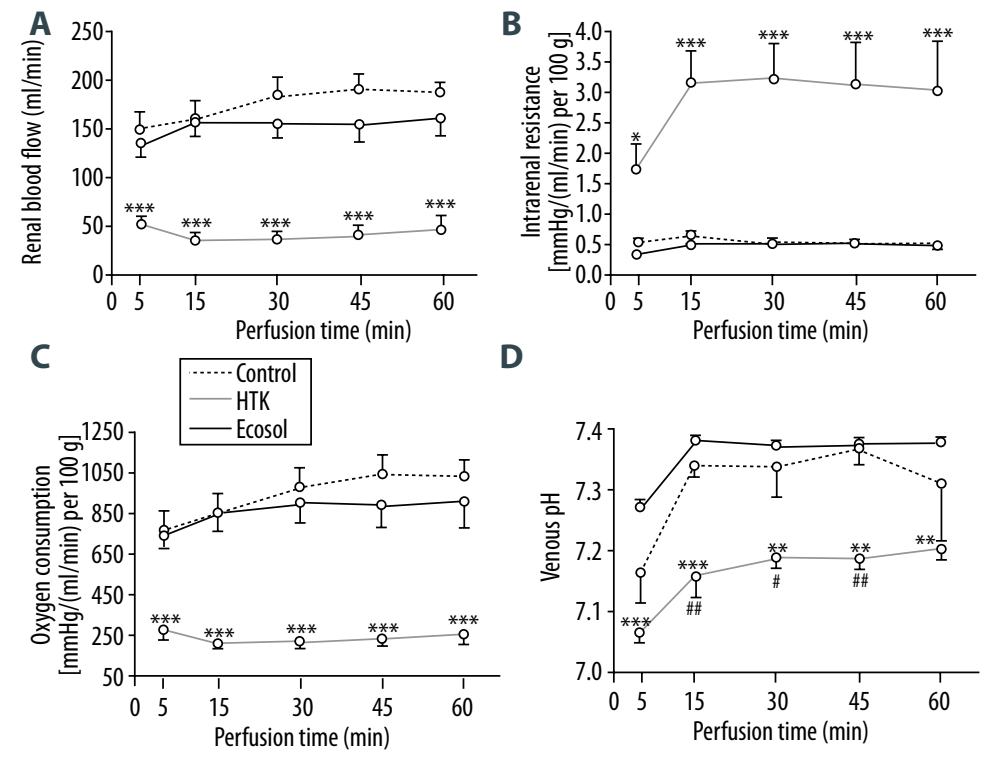

Figure 2. (A) Renal blood flow during reperfusion (Ecosol and control vs. HTK, $p<0.001$ at 5, 15, 30, 45, 60 min). (B) Intrarenal resistance per 100 $\mathrm{g}$ kidney weight during reperfusion (Ecosol and control vs. HTK, $p<0.001$ at 15, 30, 45, $60 \mathrm{~min}$; Ecosol vs. HTK, $p<0.05$ at $5 \mathrm{~min}$ ). (C) Oxygen consumption during reperfusion (Ecosol and control vs. HTK, p<0.001 at $5,15,30,45$ and $60 \mathrm{~min}$ ). (D) Venous $\mathrm{pH}$ during reperfusion (Ecosol vs. HTK, $p<0.001$ at $5,15 \mathrm{~min}, \mathrm{p}<0.01$ at 30 , 45, 60 min; HTK vs. control, $p<0.01$ at $15,45 \mathrm{~min}, \mathrm{p}<0.05$ at $30 \mathrm{~min}$ ). (A-D) 2-way ANOVA followed by Bonferroni post-test correction.
(NanoZoomer 2.0HT, Hamamatsu Photonics Deutschland GmbH, Herrsching am Ammersee, Germany). Twenty Bowman's capsules, glomerular cross-section areas and tubular outer diameters were measured per slide under 20x magnification using digital pathology system software (Hamamatsu Photonics Deutschland $\mathrm{GmbH}$ ). All analyses where done in a blinded fashion.

\section{Statistical analysis}

Statistical analysis was performed by one way analysis of variance (ANOVA) followed by Bonferroni post-test correction unless indicated otherwise, using the GraphPad Prism 5.01 software package (GraphPad Software Inc, San Diego, CA, USA) Data are presented as mean \pm SEM. Area under the curve (AUC) was calculated individually and compared using Kruskal-Wallis with Dunn's post-test. A p value $<0.05$ was considered statistically significant.

\section{Results}

\section{Kidney weight}

After recovery, kidney weights did not differ significantly (116 \pm 9 vs. $119 \pm 12$ vs. $115 \pm 8$ g; Ecosol vs. HTK vs. control respectively). During washout, kidneys in the Ecosol group lost weight in contrast to kidneys in both the HTK and control groups which gained weight, suggesting intrarenal accumulation of HTK solution ( $-15 \pm 3$ vs. $21 \pm 1$ vs. $25 \pm 3$ g respectively; Ecosol vs. HTK and control, $p<0.001)$. The time needed for washout of 500 $\mathrm{ml}$ preservation solution did not differ significantly (Ecosol vs. HTK vs. control; $18 \pm 3$ vs. $26 \pm 6$ vs. $13 \pm 1$ min respectively).

\section{Reperfusion parameters}

Renal blood flow was higher in the Ecosol group compared to the HTK group and similar to controls throughout the reperfusion period (Figure $2 \mathrm{~A}$ ). After 5 min reperfusion, the intrarenal resistance was approximately 5 -fold lower in Ecosol than in HTK and equal to controls (Figure 2B).

\section{Metabolic activity}

Metabolic activity as expressed by oxygen consumption was higher in the Ecosol group compared to HTK at all time points and similar to controls (Figure $2 \mathrm{C}$ ). Kidneys preserved in Ecosol maintained a physiological metabolic acid-base homeostasis during reperfusion in contrast to HTK, which demonstrated significantly lower venous pH levels at all time points (Figure 2D). Ecosol and controls showed comparable pH levels. At the start of reperfusion, $\mathrm{pH}$ perfusate levels were within physiological levels and equal between all groups $(7.35 \pm 0.01)$.

\section{Renal function}

Creatinine clearance was lower in the HTK group compared to controls (Figure 3A) without significant difference between Ecosol and HTK. Urinary creatinine levels were comparable between the Ecosol and HTK groups throughout the reperfusion period (AUC, $p=0.6882$ ). As a marker of tubular function, fractional excretion of sodium was higher in HTK in comparison to controls with no difference seen between Ecosol and HTK (Figure 3B). For both parameters, Ecosol did not differ significantly from the non-warm ischemia-damaged control group. Kidneys in the Ecosol group produced more urine compared to the HTK group, although the total output of urine was lower in 

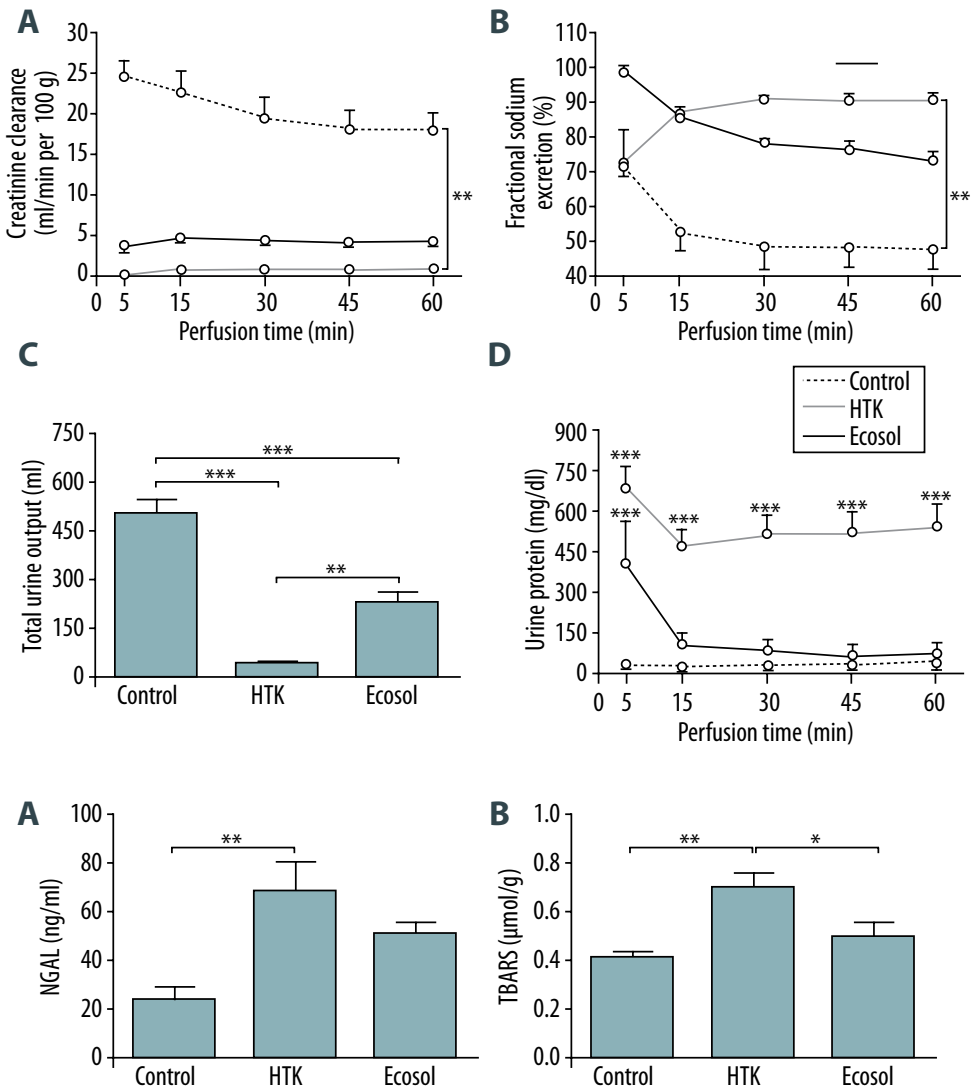

Figure 3. (A) Creatinine clearance during reperfusion (AUC: HTK vs. control, p <0.01). (B) Fractional sodium excretion during reperfusion (AUC: HTK vs. control, $p<0.01$ ). (C) Total urine output at $60 \mathrm{~min}$ (Ecosol vs. HTK, $p<0.01$; Ecosol and HTK vs. control, $p<0.001$ ). (D) Urine protein concentrations during reperfusion (Ecosol and control vs. HTK, $p<0.001$ at 5, 15, 30, 45, $60 \mathrm{~min}$; Ecosol vs. control, $p<0.001$ at $5 \mathrm{~min}$ ). (A, B) Kruskal-Wallis followed by Dunn's post-test correction. (C) One-way ANOVA followed by Bonferroni posttest correction. (D) Two-way ANOVA followed by Bonferroni post-test correction. both warm ischemia-damaged groups compared to the nonwarm ischemic controls (Figure 3C). At 5-min reperfusion, Ecosol and HTK groups demonstrated higher urinary protein concentrations compared to controls. Thereafter, urinary protein concentrations were more than 3-fold lower in Ecosol than in HTK, with Ecosol reaching values equal to controls (Figure 3D). During reperfusion, urine creatinine concentrations did not differ significantly between the Ecosol and HTK groups, with HTK being higher than controls (AUC, HTK vs. control, $\mathrm{p}<0.05$ ). Acute tubular injury was more severe in the HTK group compared to controls as expressed by higher urinary NGAL levels (Figure 4A).

\section{Oxidative status}

Reduced lipid peroxidation, as reflected by post-reperfusion tissue TBARS concentrations, was observed in Ecosol compared to HTK with Ecosol having concentrations similar to controls (Figure 4B). The GSH/GSSG ratio was higher in the Ecosol group compared to both HTK and control groups ( $4.5 \pm 0.2 \mathrm{vs}$. $1.6 \pm 0.1$ and $1.7 \pm 0.1$, respectively, $p<0.001$ ).

\section{Histology}

Macroscopic examination of kidney cross-sections after reperfusion showed no obvious pathological findings in the
Ecosol and control groups, whereas blood remnants were evident in the HTK preserved kidneys (Figure 5B). Microscopic evaluation revealed clotted blood in the microvasculature in the HTK group (Figure 6), indicating vascular occlusion resulting in increased intrarenal resistance during reperfusion. Bowman's capsule cross-section areas were enlarged in HTK compared to Ecosol and controls (Figure 7A). The same trend was observed for tubular diameter, which, however, did not reach statistical significance $(38 \pm 3$ vs. $45 \pm 3$ vs. $38 \pm 3 \mu \mathrm{m}$; Ecosol vs. HTK vs. control, respectively). Intratubular protein was observed only in the HTK group (Figure 7C), corresponding to the findings of increased urinary protein concentrations in this group.

\section{Discussion}

Kidney transplantation is the treatment of choice for endstage renal disease but is severely limited due to the present worldwide shortage of donor organs. It has been shown that increased use of DCD organs can effectively expand the donor pool [3]. Since DCD kidney grafts tolerate CS preservation less well than kidneys from brain-dead donors [30], current clinically used preservation solutions such as HTK are associated with high incidences of delayed graft function [8]. 
A

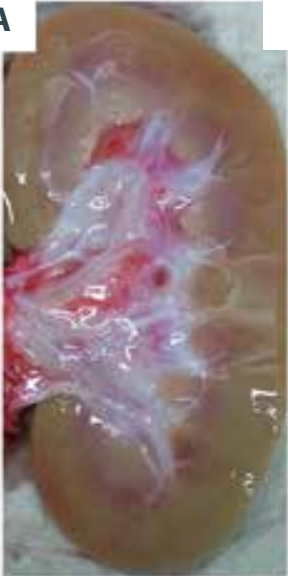

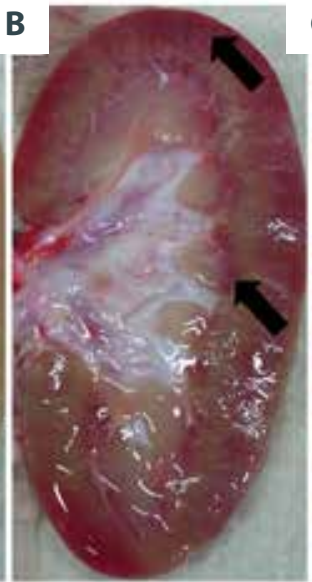

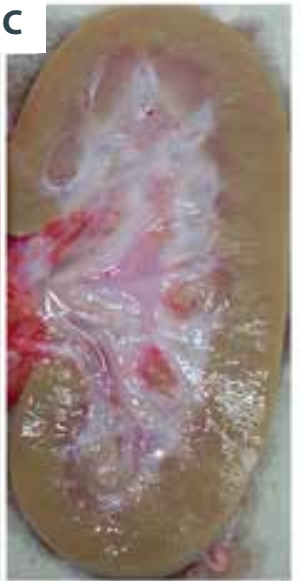

Figure 5. Kidney cross-sections after

reperfusion; (A) control, (B) HTK (black arrows: blood remnants in cortical and medullary regions), (C) Ecosol.

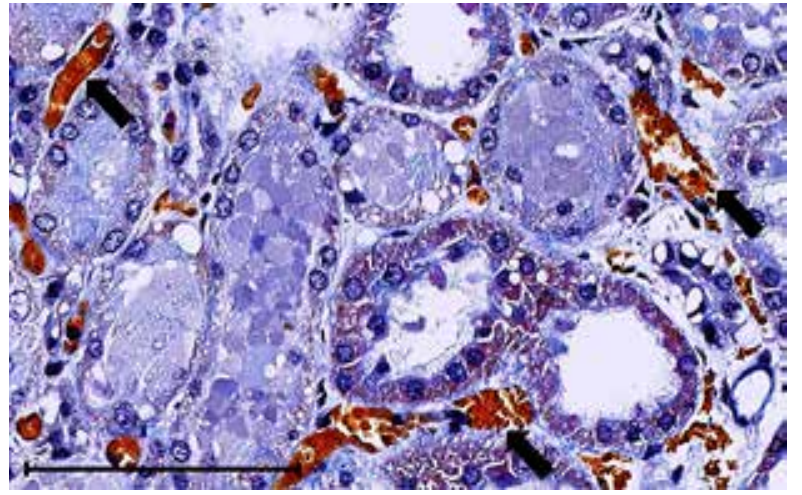

Figure 6. Microvascular blood clotting in the HTK group (AFOG stain, original magnification $\times 40$, scale bar $100 \mu \mathrm{m})$.

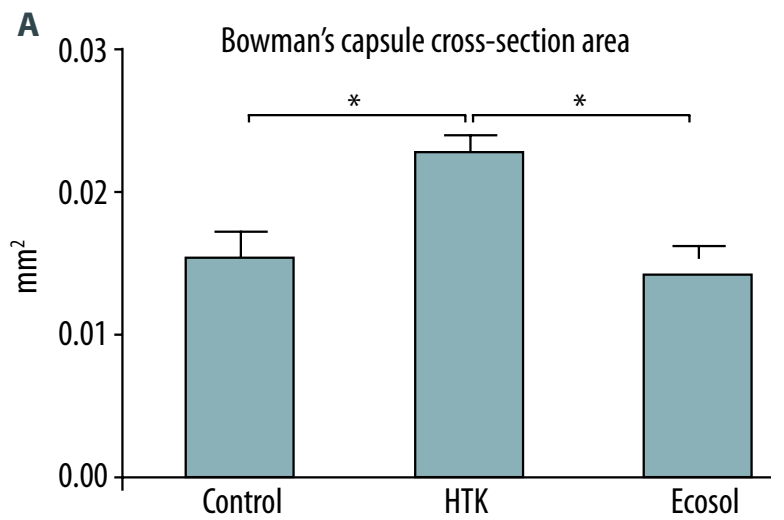

Figure 7. (A) Bowman's capsule cross-section area increased in HTK vs. control and Ecosol, p<0.01, 1-way ANOVA followed by Bonferroni post-test correction. (B) Control. (C) HTK (black arrow: enlarged Bowman's capsule space, white arrow: intratubular protein). (D) Ecosol (PAS stain, original magnification $\times 20$, scale bar 200 $\mu \mathrm{m})$.
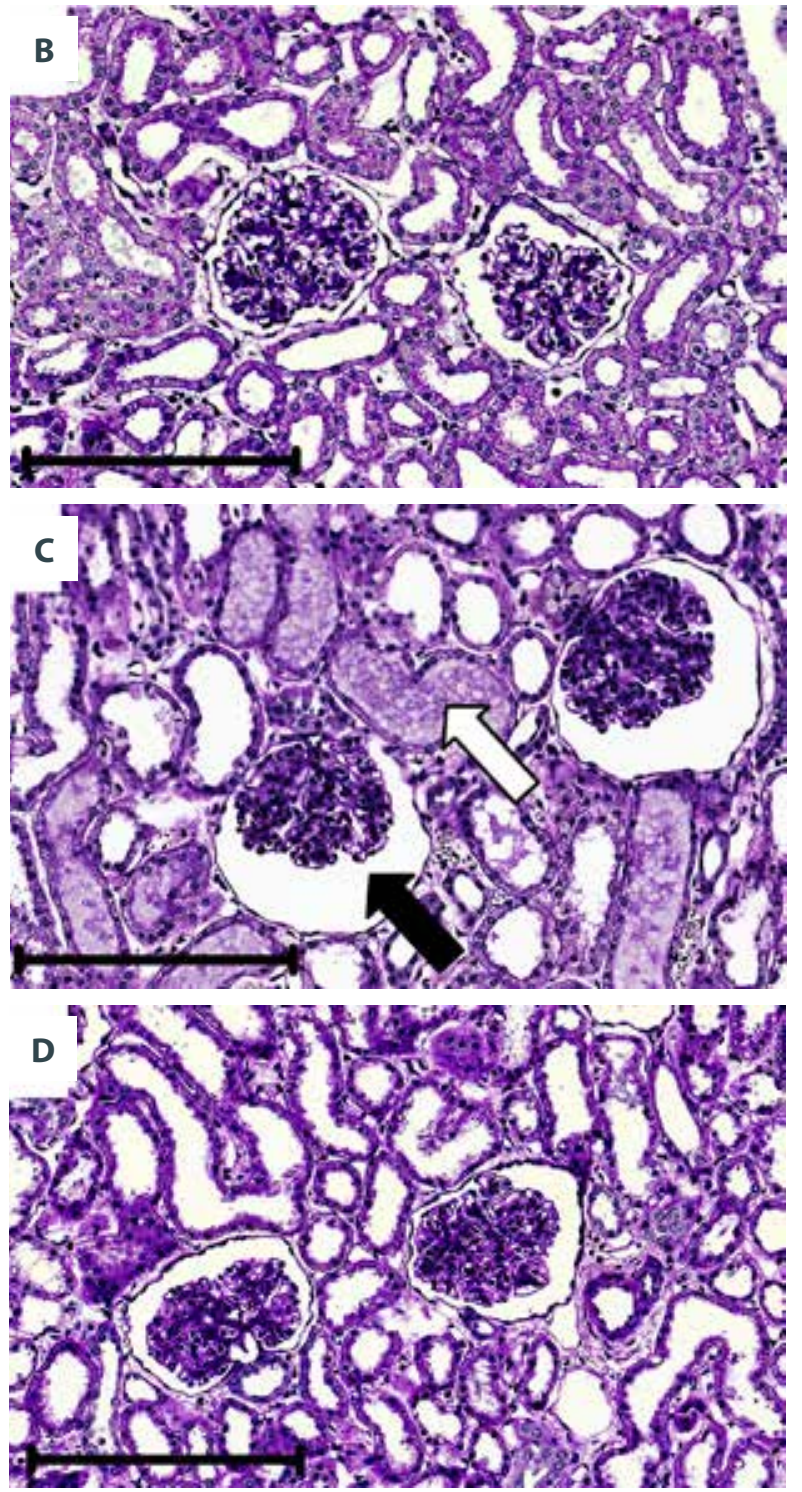
Here, we compared the novel Ecosol preservation solution with HTK solution for 24-h CS preservation of 45-min warm ischemia-damaged kidney grafts, employing non-warm ischemia damaged kidneys, cold-stored for $24 \mathrm{~h}$ in HTK as controls. The major and novel finding of this study is that the preservation quality of warm ischemia-damaged porcine kidneys coldstored in Ecosol was significantly better in comparison to HTK. In particular, Ecosol-preserved kidneys had lower intrarenal resistance, urinary protein concentrations, and reduced concentrations of selective markers of oxidative stress, and higher oxygen consumption compared to HTK. Moreover, these parameters did not differ significantly from the non-warm ischemia-damaged control group.

Successful preservation of donor organs starts with an effective washout, rapid cooling of the organ, and replacement of the donor's blood with the preservation solution [31]. Kidneys washed-out using HTK gained weight even without being subjected to warm ischemia, suggesting accumulation of the solution during washout, whereas Ecosol washout resulted in weight decrease. Although the inclusion of the colloid PEG and several impermeants in Ecosol resulted in a higher viscosity compared to HTK, the washout time was numerically lower when using Ecosol than with HTK. This is in line with a previous study showing faster washout of DCD kidneys accompanied by less remaining red blood cells in the vascular bed using a PEG and impermeants containing preservation solution, compared to HTK [23].

The development of intravascular thrombosis after cardiac arrest negatively impacts viability during consequent ischemic storage and reperfusion [32]. Moreover, warm ischemia is known to induce oxidative stress, vascular injury, and vasoconstriction, resulting in reduced renal blood flow [5]. The low intrarenal resistance in the Ecosol group, which was equal to non-warm ischemic controls from 5-min reperfusion, could be due to abrogation of the effects of warm ischemia with respect to oxidative stress and vascular injury. Further evidence for these findings was obtained by decreased TBARS concentrations in the Ecosol group compared to HTK and similar to controls. The higher levels of TBARS in the HTK group are likely attributable to ATP degradation under anoxic CS conditions leading to reactive oxygen species-mediated injury and lipid peroxidation [33]. It has been shown previously that GSH can inhibit lipid peroxidation [34] and that a high degree of inverse linear correlation exists between lipid peroxidation and GSH content [35]. Ecosol solution includes GSH, ascorbic acid, and taurine to specifically reduce oxidative stress during warm ischemia and cold anoxic preservation. The higher GSH/GSSG ratio in the Ecosol group compared to both HTK and control groups further suggests that intracellular GSH levels could be maintained during CS and subsequent reperfusion by Ecosol solution. Taurine has been shown to reduce lipid peroxidation and vascular resistance upon post-ischemic reperfusion [36]. Taurine acts as an inert osmolyte, is not metabolically active, and has a role in regulating the cell volume [37], prevention of lipid peroxidation, and reduction of proteinuria [38]. Glomerular damage and permeability to protein resulting in severe proteinuria is a known phenomenon after 24-h CS [39]. At the start of reperfusion, urinary protein concentrations were comparable between the Ecosol and HTK groups and higher than the control group. Thereafter, a steep decrease was observed in the Ecosol group to concentrations 3-fold less than HTK and similar to controls, a finding confirmed by histological examination. Since the urinary creatinine levels were comparable between Ecosol and HTK groups throughout the reperfusion period, the markedly higher urinary protein concentrations in the HTK group can be attributed to tubular damage and not to increased glomerular permeability. This hypothesis is further supported by the higher urinary levels of the acute tubular injury marker NGAL in the HTK group, compared to controls and by lower creatinine clearance, which, during isolated reperfusion, originates from proximal and distal tubular damage [40]. The presence of intratubular protein casts in the HTK group is indicative of tubular occlusion leading to pressure-induced enlargement of the Bowman's capsule space and lower urine output in the HTK group compared to Ecosol and control groups.

In the Ecosol group, oxygen consumption was higher than in the HTK group and comparable to controls, which correlated with the energy-dependent process of $\mathrm{Na}^{+}$reabsorption, as reflected by fractional excretion of $\mathrm{Na}^{+}$. Hypothermic preservation of DCD kidneys is associated with anaerobic glycolysis-induced lactate accumulation and release of lysosomal enzymes due to cell damage, leading to severe acidosis [41]. After CS of kidney grafts, HTK has been reported to cause metabolic acidosis with a pH below 6.8 [42]. In contrast to the more acidic HTK solution, Ecosol was able to maintain a physiological metabolic acid-base balance, which might be due to less cell damage as well as to the multi-buffer system in Ecosol compared to the single buffering agent, histidine, in HTK.

Ecosol was compared in this feasibility study to the widely used HTK solution since current clinically used preservation solutions have demonstrated equal clinical efficacy in the preservation of kidney grafts with HTK reported to have a cost advantage [11]. Although UW is regarded by many as the gold standard CS solution for DCD kidneys, in a recent systematic review and meta-analysis, no differences were found in the incidence of delayed graft function with the use of UW, HTK, and Celsior solution [8].

Introduced by Hemingway in 1931 [43], the well established and standardized IPPK model is limited by the absence of alloantigen reactions and in vivo follow-up, which are required to extrapolate the results to the clinical setting. However, the IPPK 
model provides a reliable and reproducible ex vivo evaluation of kidney preservation quality and assessment of new preservation methods without requiring a recipient animal. Moreover, kidneys can be used from animals used in other experiments and 2 experiments can be performed per animal, which is in accordance with the $3 R$ principle (replacement, refinement, reduction) as postulated by Russell and Burch in 1959 [44].

\section{Conclusions}

In conclusion, this study demonstrated the potential of the novel Ecosol preservation solution to improve the preservation quality of warm ischemia-damaged, cold-stored kidney grafts in comparison to HTK. Further in vivo studies involving

\section{References:}

1. National Institutes of Health: Atlas of Chronic Kidney Disease and EndStage Renal Disease in the United States. US Renal Data System Annual Data Report. 2013

2. Grams ME, Massie AB, Schold JD et al: Trends in the inactive kidney transplant waitlist and implications for candidate survival. Am J Transplant, 2013; 13(4): 1012-18

3. Abouna GM: The use of marginal-suboptimal donor organs: a practical solution for organ shortage. Ann Transplant, 2004; 9(1): 62-66

4. Neyrinck A, Van Raemdonck D, Monbaliu D: Donation after circulatory death: current status. Curr Opin Anaesthesiol, 2013; 26(3): 382-90

5. Rossard L, Favreau F, Giraud S et al: Role of warm ischemia on innate and adaptive responses in a preclinical renal auto-transplanted porcine model. J Transl Med, 2013; 11(1): 129

6. Snoeijs MG, Schaubel DE, Hene R et al: Kidneys from donors after cardiac death provide survival benefit. J Am Soc Nephrol, 2010; 21(6): 1015-21

7. Snyder RA, Moore DR, Moore DE: More donors or more delayed graft function? A cost-effectiveness analysis of DCD kidney transplantation. Clin Transplant, 2013; 27(2): 289-96

8. O'Callaghan JM, Knight SR, Morgan RD, Morris PJ: Preservation solutions for static cold storage of kidney allografts: a systematic review and metaanalysis. Am J Transplant, 2012; 12(4): 896-906

9. Gritsch HA: Transplantation: pulsatile perfusion-time for a prospective trial. Nat Rev Nephrol, 2014; 10(4): 191-92

10. Collins GM, Bravo-Shugarman M, Terasaki PI: Kidney preservation for trans portation. Initial perfusion and 30 hours' ice storage. Lancet, 1969; 2(7632): 1219-22

11. Bellamy CA, Nicely B, Mattice BJ, Teaster R: Comparative analysis of clinica efficacy and cost between University of Wisconsin solution and histidinetryptophan-ketoglutarate. Prog Transplant, 2008; 18(3): 166-71; quiz 72

12. Tillou X, Collon S, Surga N et al: Comparison of UW and Celsior: long-term results in kidney transplantation. Ann Transplant, 2013; 18: 146-52

13. O'Callaghan JM, Morgan RD, Knight SR, Morris PJ: Systematic review and meta-analysis of hypothermic machine perfusion versus static cold storage of kidney allografts on transplant outcomes. Br J Surg, 2013; 100(8): 991-1001

14. Voigt MR, DeLario GT: Perspectives on abdominal organ preservation solutions: a comparative literature review. Prog Transplant, 2013; 23(4): 383-91

15. Grat $M$, Ligocka J, Lewandowski $Z$ et al: Incidence, pattern and clinical relevance of microbial contamination of preservation fluid in liver transplantation. Ann Transplant, 2012; 17(3): 20-28

16. Ben Abdennebi $\mathrm{H}$, Steghens JP, Hadj-Aissa A et al: A preservation solution with polyethylene glycol and calcium: a possible multiorgan liquid. Transp Int, 2002; 15(7): 348-54

17. Wada $\mathrm{H}$, Liu CJ, Yokomise $\mathrm{H}$ et al: Newly developed preservation solution for the lung: 30-hour preservation with new ET-Kyoto solution. Transplant Proc, 1996; 28(3): 1178-80 allotransplant models and comparison to other clinically applied preservation solutions are warranted.

\section{Acknowledgments}

The authors would like to thank Thaddeus Stopinski, Dr. med. vet. Anna Woitok, Renate Nadenau, Birgit Nellessen, Sabrina Heblik, Dr. Ali Kashefi and Regina Ben Hamza for their continuous support of the study.

\section{Disclosure}

$B M D$ is an inventor of the patent. BMD and RHT are shareholders of TX Innovations BV. All other authors have no conflict of interest.
18. Billault C, Vaessen C, Van Glabeke E et al: Use of the SCOT solution in kidney transplantation: preliminary report. Transplant Proc, 2006; 38(7): 2281-82

19. Schreinemachers MC, Doorschodt BM, Florquin S et al: Pulsatile perfusion preservation of warm ischaemia-damaged experimental kidney grafts. B J Surg, 2010; 97(3): 349-58

20. Belzer FO, Southard JH: Principles of solid-organ preservation by cold storage. Transplantation, 1988; 45(4): 673-76

21. Churchill TA, Green CJ, Fuller BJ: Protective properties of amino acids in liver preservation: effects of glycine and a combination of amino acids on anaerobic metabolism and energetics. J Hepatol, 1995; 23(6): 720-26

22. Churchill TA, Kneteman NM: Investigation of a primary requirement of organ preservation solutions: supplemental buffering agents improve hepatic energy production during cold storage. Transplantation, 1998; 65(4): 551-59

23. Schreinemachers MC, Doorschodt BM, Florquin S, Tolba RH: Comparison of preservation solutions for washout of kidney grafts: an experimental study. Transplant Proc, 2009; 41(10): 4072-79

24. Giraud S, Codas R, Hauet T et al: Polyethylene glycols and organ protection against I/R injury. Prog Urol, 2014; 24(Suppl.1): S37-43

25. Schreinemachers MC, Doorschodt BM, Florquin S et al: Improved preservation and microcirculation with POLYSOL after transplantation in a porcine kidney autotransplantation model. Nephrol Dial Transplant, 2009; 24(3) 816-24

26. Yagi S, Doorschodt BM, Afify $M$ et al: Improved preservation and microcirculation with POLYSOL after partial liver transplantation in rats. J Surg Res, 2011; 167(2): e375-83

27. Toledo-Pereyra LH: Organ preservation for transplantation. $3^{\text {rd }}$ edition. Landes Bioscience, 2010: 1-15

28. Arana C, Cutando A, Ferrera MJ et al: Parameters of oxidative stress in saliva from diabetic and parenteral drug addict patients. J Oral Pathol Med, 2006; 35(9): 554-59

29. Behuliak M, Palffy R, Gardlik R et al: Variability of thiobarbituric acid reacting substances in saliva. Dis Markers, 2009; 26(2): 49-53

30. Summers DM, Johnson RJ, Hudson A et al: Effect of donor age and cold storage time on outcome in recipients of kidneys donated after circulatory death in the UK: a cohort study. Lancet, 2013; 381(9868): 727-34

31. $t$ Hart NA, van der Plaats A, Leuvenink HG et al: Initial blood washout dur ing organ procurement determines liver injury and function after preservation and reperfusion. Am J Transplant, 2004; 4(11): 1836-44

32. Kuroe K, Kurokawa T, Nishikimi $M$ et al: Effects of thromboxane A2 synthetase inhibitor on postischemic liver injury in rats. Eur Surg Res, 1991; 23(1): 20-26

33. Devasagayam TP, Boloor KK, Ramasarma T: Methods for estimating lipid peroxidation: an analysis of merits and demerits. Indian J Biochem Biophys, 2003; 40(5): 300-8 
34. Christophersen BO: The inhibitory effect of reduced glutathione on the lipid peroxidation of the microsomal fraction and mitochondria. Biochem J, 1968; 106(2): 515-22

35. Maddaiah VT: Glutathione correlates with lipid peroxidation in liver mitochondria of triiodothyronine-injected hypophysectomized rats. FASEB J, 1990; 4(5): 1513-18

36. Lauschke $H$, Kotting $M$, Akbar S, Minor T: Use of taurine as antioxidant in resuscitating livers from non-heart-beating donors by gaseous oxygen persufflation. J Invest Surg, 2003; 16(1): 7-11

37. Chesney RW, Han X, Patters AB: Taurine and the renal system. J Biomed Sci, 2010; 17(Suppl.1): S4

38. Trachtman H, Futterweit S, Maesaka J et al: Taurine ameliorates chronic streptozocin-induced diabetic nephropathy in rats. Am J Physiol, 1995; 269(3 Pt 2): F429-38

39. Lambert R, Henry M, Howden B et al: Glomerular damage after kidney preservation. Transplantation, 1986; 42(2): 125-30
40. Khan TN, Sinniah R: Morphometric study showing the importance of distal tubular damage in impaired creatinine clearance. Am J Nephrol, 1993 13(3): 178-83

41. van den Eijnden MM, Leuvenink HG, Ottens PJ et al: Effect of brain death and non-heart-beating kidney donation on renal function and injury: an assessment in the isolated perfused rat kidney. Exp Clin Transplant, 2003; 1(2): 85-95

42. Dolinska B, Ostrozka-Cieslik A, Caban A et al: Comparing the effect of Biolasol(R) and HTK solutions on maintaining proper homeostasis, indicating the kidney storage efficiency prior to transplantation. Ann Transplant, 2012; 17(2): 74-78

43. Hemingway A: Some observations on the perfusion of the isolated kidney by a pump. J Physiol, 1931; 71(2): 201-13

44. Russell W.M.S. BRL. The Principles of Humane Experimental Technique. Methuen, London, 1959 